\section{Changing incidence of non-accidental injury to children in South Glamorgan}

The increasing number of reports in the media of severe or sometimes fatal non-accidental injury to children suggests that the incidence of this problem may have increased. A previous paper examined the incidence of child abuse in South Glamorgan from 1970 to $1981,{ }^{1}$ and this report is an update of that study.

\section{Methods and results}

We examined details of all referrals during 1982-5 in which non-accidental injury had been diagnosed. The procedure for child abuse in South Glamorgan requires that all children who are suspected of having suffered non-accidental injury are medically examined. All children with suspected or proved injury are admitted to hospital. Within 72 hours after referral there is a case conference, which is attended by a senior paediatrician. After considering medical reports and contributions from other agencies those at the case conference decide whether a diagnosis of non-accidental injury can be upheld. This process of reaching a diagnosis of non-accidental injury has not changed appreciably during the past 15 years.

The data for this report were taken from the minutes of the case conferences; copies of all these minutes are received and monitored centrally by the child abuse coordinator (JJ) for South Glamorgan Social Services. The age range of the children was 0-15 years. Injuries were divided into two groups: severe injuries, which included all fractures, internal injuries, and intracranial bleeding, and moderate injuries, which included all soft tissue injuries.

The figure shows a rapid increase in the number of children referred with moderate injuries after 1973. In 1974 there was a ninefold increase in the number of children aged 0-4 years who were referred with moderate injuries; subsequently an average of 22 children in this age group were referred each year. The number of children aged 5-9 who were referred with moderate injuries increased from four in 1974 to 18 in 1975; after 1974 there was an average of 15 referrals each year. The referral of children aged 10-15 with moderate injuries was a later phenomenon: during 1970-7 the average number of cases referred each year was 1.9 but after 1977 the average number of referrals increased to 13 , showing that child abuse is no longer a syndrome affecting only infants.

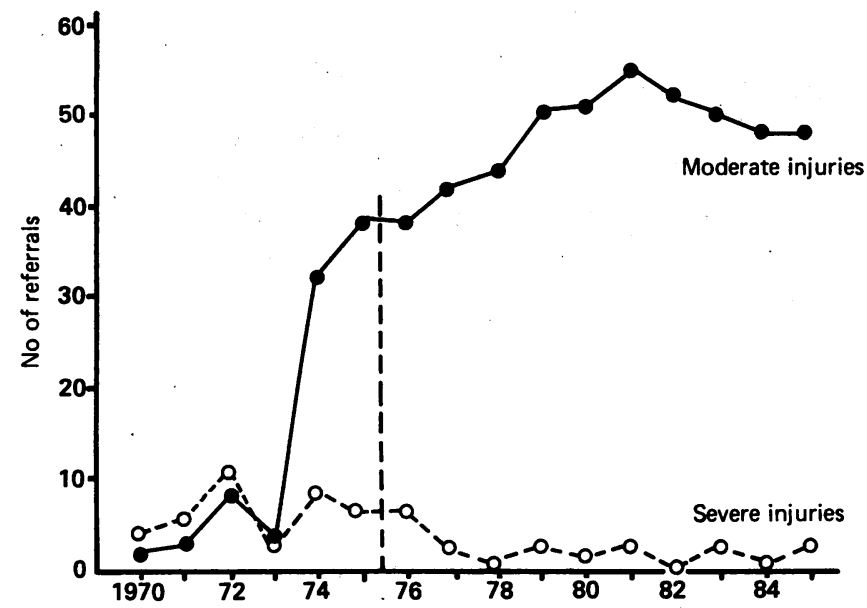

Incidence of non-accidental injury to children, 1970-85. Vertical broken line indicates when child abuse procedures were introduced.

\section{Comment}

The report of the inquiry into the killing of Maria Colwell by her stepfather was published in $1974 .^{2}$ It was followed by a circular from the Department of Health and Social Security that urged local authorities to set up area review committees to be responsible for devising local multidisciplinary procedures and setting up child abuse registers. ${ }^{3}$ In South Glamorgan the existing procedures were revised and were circulated to many more people than before. Many training programmes were instigated for all those working in the care of children, with an emphasis on increasing the awareness of the problem and recognising and acting on warning signs. This resulted in earlier intervention, and the sharp rise in the number of referrals for moderate injuries occurred at this time. This rise therefore seems to have been due to the combination of an emphasis on publicity, improved management, and increased training in child abuse. Teachers, in particular, participated for the first time in training and procedures, which explains most of the increase in the number of referrals of school aged children. The drop in the number of children referred with severe injuries in the mid-1970s was sustained. One explanation for this might be the increased reporting of children with suspected or proved moderate non-accidental injuries; this ensures that treatment begins before a more serious injury occurs and gives an opportunity for effective preventive work.

1 Jenkins J, Gray OP. Changing clinical picture of non-accidental injury to children. $\mathrm{Br} \mathrm{Med} \mathcal{J}$ 1983;287:1767-9.

Department of Health and Social Security. Report of the committee of inquiry into the care and supervision provided in relation to Maria Colwell. London: DHSS, 1974.

3 Department of Health and Social Security. Non-accidental injury to children. London: DHSS, 1974 (CMO 3/74.)

(Accepted 26 March 1987)

South Glamorgan Social Services Department, Cardiff CF1 3BY

JANE JENKINS, MA, MSC, child abuse coordinator

Department of Child Health, University of Wales College of Medicine, Cardiff CF4 4XN

O P GRAY, FRCP, DCH, professor

Correspondence to: Mrs Jenkins.

\section{Fatal bronchospasm after topical lignocaine before bronchoscopy}

Fibreoptic bronchoscopy has a low morbidity and mortality. We report the first case of death due to hypersensitivity to topical local anaesthetic (lignocaine; $10 \%$ Xylocaine spray) given during routine premedication for this procedure.

\section{Case report}

A 67 year old man with chronic bronchitis and emphysema presented with increasing dyspnoea and weight loss. He had severe airflow obstruction (forced expiratory volume in one second $0.5 \mathrm{l}$, forced vital capacity $1.7 \mathrm{l}$ ). A chest radiograph was compatible with the diagnosis of emphysema but showed no suggestion of a carcinoma. An electrocardiogram showed sinus rhythm with occasional ventricular extrasystoles. Arterial oxygen tension was $7.6 \mathrm{kPa}$ and arterial carbon dioxide tension $6.4 \mathrm{kPa}$ when he was breathing air. Because of the weight loss bronchoscopy was arranged to exclude carcinoma.

He was admitted fasting and given nebulised salbutamol $(5 \mathrm{mg})$ one hour before bronchoscopy and atropine $(0.6 \mathrm{mg})$ intravenously as premedication without adverse effect. Two puffs of lignocaine $(20 \mathrm{mg})$ were administered to one nostril and six puffs $(60 \mathrm{mg})$ to the posterior pharyngeal wall. Two minutes later he developed wheezy dyspnoea and cyanosis, which were not relieved by inhaled salbutamol, and one minute later he suffered a cardiorespiratory arrest. External cardiac massage was started with manual ventilation through a face mask and oropharyngeal airway and subsequently a $7.5 \mathrm{~mm}$ endotracheal tube inserted by an anaesthetist. There was no evidence of laryngospasm, although manual ventilation was difficult, possibly because of severe bronchospasm. There was no evidence of aspiration of stomach contents. Sodium bicarbonate $8.4 \%$ solution $(100 \mathrm{ml}$ ) was infused followed by $10 \mathrm{ml}$ of $1 / 1000$ adrenaline and $10 \mathrm{ml}$ calcium chloride. This produced ventricular fibrillation; cardioversion ( 340 joules) and an intravenous bolus of $100 \mathrm{mg}$ of lignocaine resulted in asystole. Despite treatment with intracardiac adrenaline and calcium chloride, isoprenaline $200 \mu \mathrm{g}$, and two further doses of adrenaline he remained asystolic. Twenty minutes after the onset of cardiorespiratory arrest attempts at resuscitation were stopped.

Necropsy confirmed the presence of a left sided pneumothorax with extensive centrilobular emphysema. Microscopy showed hypertrophy of smooth muscle and thickening of the basement membrane in the small airways.

\section{Comment}

Although we suspected that this man died of a hypersensitivity reaction to topical lignocaine, other factors had to be considered: atropine could have precipitated a ventricular arrhythmia, although his condition did not deteriorate until 12 minutes after its administration, tachycardia was not observed beforehand, and he was asystolic. The pneumothorax could have been due to the cardiac needle, cardiac massage, or manual ventilation in the presence of emphysema but is unlikely to have been an initiating factor for this event. In view of the short interval between the use of lignocaine spray and the onset of wheezing we concluded that the patient died of a hypersensitivity reaction to the local anaesthetic.

A survey of the complications of 24000 fibreoptic bronchoscopies gave the incidence of major complications as $0.08 \%$ with a mortality of $0.01 \%{ }^{1}$ No deaths were due to lignocaine, although one patient died of seizures after excessive use of local anaesthetic spray.

The manufacturers of lignocaine recommend a maximum dose of $200 \mathrm{mg}$ for a $70 \mathrm{~kg}$ man. Our patient weighed $45 \mathrm{~kg}$ and was given a total of $80 \mathrm{mg}$. 
This plus the immediacy of the reaction suggest that his death was unlikely to have been due to toxicity.

Nebulised lignocaine may cause narrowing of the airways in patients with asthma, ${ }^{2}$ but this is rarely of clinical importance especially as nebulised bronchodilators are given before fibreoptic bronchoscopy. Hypersensitivity to lignocaine is uncommon. Urticarial reactions may occur, ${ }^{3}$ and deaths due to anaphylaxis after small doses of injected local anaesthetic have been reported..$^{45}$ We believe that this is the first reported death due to topical lignocaine during fibreoptic bronchoscopy. This procedure, however, remains safe with few complications.

1 Credle W, Smidy J, Elliott RC. Complications of fibreoptic bronchoscopy. Am Rev Respir Dis 1974;109:67-72.

2 Weiss EB, Patwardhan AV. The response to lidocaine in bronchial asthma. Chest 1977;72:429-38. 3 Waldman HB, Binkley C. Lignocaine hypersensitivity: report of a case. $f$ Am Dent Assoc 1967;74:747-9.

4 Morisset LM. Fatal anaphylactic reaction to lidocaine. Amed Forces Medical fournal 1957;8:740-4.

5 Kirkler DM. Allergy to lignocaine. Lancet 1962;i:159.

(Accepted 11 March 1987)

Department of Respiratory Medicine, East Birmingham Hospital, Birmingham B9 5ST

S P RUFFLES, MRCP, MRCGP, registrar

J G AYRES, MD, MRCP, consultant physician

Correspondence to: Dr Ruffles.

\section{Minimising bruising in the antecubital fossa after venepuncture}

Because of the large number of bruises seen after venepuncture of the antecubital fossa in patients presenting for anaesthesia we investigated the phenomenon.

\section{Methods and results}

We recruited 20 volunteers (eight men and 12 women) aged $20-47$ with blood pressures of less than $150 / 95 \mathrm{~mm} \mathrm{Hg}$. Each had $5 \mathrm{ml}$ of blood taken from both antecubital fossas by means of a green, 21 gauge Monoject needle and a tourniquet inflated to exactly half the diastolic blood pressure to promote venous congestion. After deflation of the tourniquet haemostasis was promoted by one of two methods based on our observations of current ward practice. In the first method the subjects pressed the contralateral thumb on to a cotton wool ball ove the site of venepuncture, keeping the elbow extended. In the second method the subject flexed the elbow, keeping the cotton wool ball over the puncture site. Both methods were used for two minutes, and the site was then inspected for 15 seconds. If bleeding had not stopped the method was repeated for one minute with the same inspection procedure; this was repeated until there was no visible bleeding. An adhesive dressing was then applied.

Subjects were randomised for which arm was to be flexed or extended and thus served as their own controls. All venepunctures were performed by the same investigator. A criterion of the study was that subjects with "difficult veins" and more than one clean puncture of the vein were excluded, as were those who had recently taken aspirin; in practice there were no exclusions. After 48 hours a second investigator, who was unaware of which method of haemostasis had been used, examined the venepuncture sites. Any visible bruising was noted and the area measured with tracing paper and a planimeter; the subject was asked about any symptoms associated with the bruising.

Flexing of the elbow after venepuncture produced visible bruising in 15 subjects compared with only six when the arm was held straight $(p<0.05$ by McNemar's test for paired data). In addition, the area of bruising was greater in the elbows that were flexed (median $0.55 \mathrm{~cm}^{2}$ and maximum $4.88 \mathrm{~cm}^{2}$ compared

Bruising 48 hours after venepuncture using two different methods to promote haemostasis

\begin{tabular}{lccc}
\hline & Total & $\begin{array}{c}\text { No } \\
\text { bruised }\end{array}$ & $\begin{array}{c}\text { Median (range) } \\
\text { area of bruise }\left(\mathrm{cm}^{2}\right)\end{array}$ \\
\hline $\begin{array}{l}\text { Elbow flexed: } \\
\text { Left arm }\end{array}$ & 10 & 8 & $0 \cdot 56(0-3 \cdot 62)$ \\
$\begin{array}{l}\text { Right arm } \\
\text { Elbow straight: }\end{array}$ & 10 & 7 & $0 \cdot 39(0-4 \cdot 88)$ \\
Left arm & 10 & 3 & $0(0-4 \cdot 84)$ \\
Right arm & 10 & 3 & $0(0-1 \cdot 84)$ \\
\hline
\end{tabular}

with median $0 \mathrm{~cm}^{2}$ and maximum $4.84 \mathrm{~cm}^{2}$ in the elbows that were extended; $\mathrm{p}<0.01$ by Wilcoxon's test for paired differences).

Thirty sites showed no visible bleeding after two minutes. One venepuncture site bled for over five minutes, but all the rest had stopped bleeding by 3 minutes 15 seconds. The time for bleeding to stop was unrelated to the size of the subsequent bruise $(p=0 \cdot 1$, Spearman's rank correlation coefficient).

\section{Comment}

We conclude that the common practice of flexing the elbow after venepuncture is not an efficient way of preventing bruising in the antecubital fossa.

We thank Professor W Mapleson for his kind help.

(Accepted 26 March 1987)

Department of Anaesthetics, University Hospital of Wales, Cardiff CF4 4XW A DYSON, MB, FFARCS, registrar in anaesthetics D BOGOD, MB, FFARCS, senior registrar in anaesthetics

Correspondence to: Dr Bogod.

\section{Huntington's disease: deterioration in clinical state during treatment with angiotensin converting enzyme inhibitor}

We report on a woman with Huntington's disease whose condition deteriorated dramatically during treatment with the angiotensin converting enzyme inhibitor captopril and improved on withdrawal of the drug.

\section{Case report}

A 47 year old woman who had been diagnosed eight years earlier as suffering from Huntington's disease was admitted to hospital for control of newly diagnosed hypertension $(210 / 120 \mathrm{~mm} \mathrm{Hg})$. She had previously been managed at home with haloperidol $1.5 \mathrm{mg}$ thrice daily. On examination she walked without help and had typical features of Huntington's disease, with diffuse involuntary choreiform movements, facial apraxia, and inability to protrude her tongue for longer than five seconds. Her higher mental functions were impaired, although she could communicate and follow simple commands. She was otherwise medically well and had no clinical features of end organ damage due to hypertension.

Haloperidol was continued and captopril $18.75 \mathrm{mg}$ daily in divided doses started. Because her blood pressure was unresponsive the dose was increased and over five days she received a total of $468 \cdot 75 \mathrm{mg}$. Her clinical state then deteriorated and she became bedbound and totally unable to communicate and showed generalised rigidity and incontinence. She did not recognise her family and required nasogastric feeding. At no stage had she had any hypotensive episode, metabolic derangement, or fever. Her blood pressure remained raised throughout, with systolic pressure varying between 220 and $180 \mathrm{~mm} \mathrm{Hg}$ and diastolic pressure varying between 150 and $120 \mathrm{~mm} \mathrm{Hg}$. Extensive investigations including lumbar puncture, thyroid function tests, and computed tomography of the brain showed no cause for her altered neurological state. Captopril was stopped and her blood pressure eventually controlled with a combination of nifedipine, atenolol, and a thiazide diuretic. Her neurological state gradually improved, and five days after the captopril was stopped she had recovered her previous functional state. Her blood pressure was controlled, and she was discharged home.

\section{Comment}

Altered amounts of various neurotransmitters have been shown in the striatonigral system of patients with Huntington's disease, ${ }^{1}$ although the underlying biochemical defect that causes the disease is unknown. In a necropsy study Arregui et al showed that the corpus striatum and substantia nigra of patients with Huntington's disease had diminished quantities of angiotensin converting enzyme. ${ }^{2}$ This enzyme has been localised to the striatonigral pathway of rat brains in autoradiographic experiments with tritiated captopril. ${ }^{3}$

This patient's pronounced deterioration during treatment with captopril for hypertension and her subsequent recovery after withdrawal of the drug suggest that inhibition of angiotensin converting enzyme was the cause of her altered neurological state. This observation may have important 